\title{
Consumer Behavior in Low Involvement Product Purchase: A Stochastic Model
}

\author{
Atanu Adhikari \\ Indian Institute of Management Kozhikode (IIMK), Kozhikode, India \\ Email: atanu.adhikari@iimk.ac.in
}

How to cite this paper: Adhikari, A. (2019) Consumer Behavior in Low Involvement Product Purchase: A Stochastic Model. Theoretical Economics Letters, 9, 424-430.

https://doi.org/10.4236/tel.2019.92030

Received: November 12, 2018

Accepted: February 24, 2019

Published: February 27, 2019

Copyright $\odot 2019$ by author(s) and Scientific Research Publishing Inc. This work is licensed under the Creative Commons Attribution International License (CC BY 4.0).

http://creativecommons.org/licenses/by/4.0/

(c) (i) Open Access

\begin{abstract}
Consumers provide less time and collect less information in buying decision of low involvement products. Consequently, they engage little thought process in their purchase decision. This is primarily because low involvement products are often low priced and carry low cost of failure. Along with uncertainties, in many situations, particularly in low involvement products and frequently purchased consumer packaged goods, little conscious decision making takes place. In such situations stochastic model-concentrating on random nature of choice becomes more appropriate than deterministic approach. In this research, we develop a stochastic model for consumer buying decision of low involvement products. We have considered agitations a buyer experience during their purchase occasion. These agitations create internal force that stimulates consumer mind. These forces are chaotic, and so the resultant force which makes purchase decision random.
\end{abstract}

\section{Keywords}

Consumer Behavior, Low Involvement

\section{Introduction}

Consumer Purchase Decision of low involvement products often involve very little thought process, information gathering and proper decision making. For example, buying a pack of chewing gum or chocolate while checking out in a retail store barely takes more than a few seconds as these products are low involvement and consumers buy these products on their impulses. This is primarily because low involvement products are often low priced and carry low cost of failure. Information available to take a buying decision of such products may not strongly guide a purchaser while taking buying decision. Previous researches suggested multiple approaches in modelling consumer purchase behaviour of 
low involvement products. Along with uncertainties, in many situations, particularly in low involvement products and frequently purchased consumer packaged goods, little conscious decision making takes place. In such situations stochastic model-concentrating on random nature of choice becomes more appropriate than deterministic approach. Another reason that stochastic choice model is suitable for such goods is availability of large volume of brand switching data with market researchers [1].

We assume that stochastic purchase behavior observed in the consumers is due to some kind of agitation within the consumers before or during the purchase. Due to such agitation in the mind of the consumers, purchase decision of above products experience several internal forces in different directions. Consequently, these forces imbalance consumers' mind and purchase decision become random. Since these forces are haphazard, resultant force that influences the purchase decision is also haphazard. The smaller the involvement, the larger is the resultant force and consequently more irregular the movements are.

This study contributes to marketing literature by modelling consumer's choice behaviour of low involvement product mapping consumer's mind with movement of gaseous molecules. There is no study available in consumer behaviour literature that has conceived movement of consumer mind with respect to stochastic agitation and consequently their decision making.

\section{Literature Review}

Research on consumer Available in marketing literature since 1963. Roberts and Lattin [2] provided systemize catalogue of consumers consideration set. In addition to that there are several research done to investigate consumer behaviour in considering different brands while taking a buying decision. Research done by DeSarbo, Young and Rangasswamy [3] proposed stochastic multidimensional unfolding to represent the consideration set.

Several other researchers have considered Bernoulli model to captcha heterogeneity in stochasticity of consumer behaviour. Other researchers have considered Markov process to do the same. The independent and dependent probability diffusion process was investigated and mathematical models for marketing share calculation through Markov chain was developed. Subsequent research also investigated linear model to predict consumer behaviour. Subsequent research also explode stochastic consumer behaviour model for market penetration of different varieties. Many researchers have taken this models one step farther by generalizing through empirical validation.

Mathematical models on customer retention has also been studied in consumer behaviour literature. Gupta and Zeithaml [4], define customer retention as the probability of customer being alive or repeat buying from a firm. Other researchers have also developed various models of retention [5].

However, the above researchers have not investigated consumer behaviour in the context of random agitation in consumer's mind at the place of purchase, 
and have not modelled how such behaviour takes place. This research tries to bridge that gap in marketing literature of consumer behaviour.

\section{The Model}

The basic concept on which kinetic theory stands is the motion of molecules due to thermal agitation. Although kinetic theory explains many thermal and allied phenomena, direct experimental evidence of random motion of molecules came only after the theory of Robert Brown and termed as Brownian motion. In 1900, Bachelier exhibited Markovian nature of Brownian motion. According to such phenomenon position of a particle at time $(t+s)$ depends on its position at time $t$ and does not depend on its position before time $t$. Markov model in stochastic consumer choice behavior assumes impact of just previous purchase on present purchase. This can be extended to post purchase behavior of the consumer for next purchase. Hence this assumption is similar to Bachelier's exhibition of Markovian nature of Brownian motion.

This paper introduces and implements mathematical approach of Brownian motion in consumers' purchase decision $(D t)$ in low involvement product. We assume here that this kind of stochastic process is a stochastic process of continuous time $\{D t: 0 \leq t<T\}$. This means that it is a standard Brownian motion on $(0, T)$ if it has the following properties.

1) $D_{0}=0$

2) The increments of $D t$ are independent: that is for any finite sets of times. $0 \leq t_{1}<t_{2}<t_{3} \cdots<t_{n}<T$ then the random variables

$D_{t 2}-D_{t 1}, D_{t 3}-D_{t 2}, D_{t 4}-D_{t 3}, \cdots, D_{t n}-D_{t(n-1)}$ are independent.

3) For any $0 \leq c<t<T$, the increment $D_{t}-D_{c}$ has a Gaussian distribution with mean zero and variance $(t-c)$.

4) For all $\alpha$ in a set of probability one, $\mathrm{D}_{t}(\alpha)$ is a continuous function of " $t$ ".

The main purpose of this article is to show that one can represent consumers' purchase behavior of low involvement and frequently purchased products as Brownian motion. In other words, we are to prove the existence of the process by conforming above four defined properties of Brownian motion.

With a view to provide support to our hypothesis that construction of Brownian motion is true, we are required to use multivariate Gaussian distribution. The most critical factor of multivariate Gaussian is that their joint density is fully determined by mean vector and variance-covariance matrix [6]. Hence we are required to identify tools that help us check that agitations of our processes are Gaussian distribution as well as the agitations are independent. As per the properties of multivariate Gaussian, if $B$ is a d-dimensional random vector, then

$$
\text { Mean vector of } B=\left[\begin{array}{c}
B_{1} \\
B_{2} \\
\cdots \\
B_{d}
\end{array}\right] \text { is a vector } \mu=\left[\begin{array}{c}
\mu_{1} \\
\mu_{2} \\
\mu_{3} \\
\cdots \\
\mu_{d}
\end{array}\right]
$$


And the varience-covarience matrix of $B$ is given by

$$
\begin{gathered}
\sum=\left[\begin{array}{c}
\sigma_{11} \sigma_{12} \sigma_{13} \cdots \sigma_{1 d} \\
\sigma_{21} \sigma_{22} \sigma_{23} \cdots \sigma_{2 d} \\
\sigma_{31} \sigma_{32} \sigma_{33} \cdots \sigma_{3 d} \\
\cdots \\
\cdots \\
\sigma_{d 1} \sigma_{d 2} \sigma_{d 3} \cdots \sigma_{d d}
\end{array}\right] \\
\sigma_{i j}=E\left[\left(B_{i}-\mu_{i}\right)\left(B_{i}-\mu_{i}\right)\right]
\end{gathered}
$$

and

$$
f(B)=\frac{1}{\sqrt[d]{2} \pi}\left[\exp \left\{-\frac{1}{2}(x-\mu)^{\mathrm{T}} \Sigma^{-1}(x-\mu)\right\}\right] \text {, for all } x \in R^{d}
$$

The profile of Brownian motion in this case can be represented as at each instant in time, the consumer randomly chooses a brand and then purchases that brand. This approach is both intuitive and rigorous [7]. We assume each time of purchase is a combination of several decisions taken at multiple of $\Delta t$. In each of such instant, the consumer randomly chooses to buy or not to buy one brand and goes to second. We can express that if a consumer starts from a particular point, in every $\Delta t$ time it covers a distance of $\Delta x$ from starting point. To model this randomness, we consider a sequence of identically distributed random variables $\left(\mathrm{Y}_{\mathrm{i}}, i>1\right)$ such that $P\left(Y_{i}=\Delta x\right)=P\left(Y_{i}=-\Delta x\right)=1 / 2$.

At time $t$, the purchaser to make $[t / \Delta t]$ moves (where $[\mathrm{g}]$ denotes the integer part of $\mathrm{g}$ ). The respondents position will be $U_{t}=Y_{1}+Y_{2}+Y_{3}+Y_{4}+\cdots+Y_{[t / \Delta t]}$. All this takes place on a very small scale at the time of purchase or just before purchase. We would like to assume both $\Delta t$ and $\Delta x$ tend to zero in an appropriate way. Note that $E U^{2} \approx(\Delta x)^{2} \cdot(t / \Delta t)$. In order for this expression to have a limit, we must consider that $(\Delta x)^{2} / \Delta t$ have a limit. The increment $\Delta t$ will be very small and $\Delta x$ will also be small so that $(\Delta x)^{2}$ will also be very small. The most appropriate choice is $\Delta x=(\Delta t)^{1 / 2}$ and $\Delta t=1 / n$, where " $n$ " is integer.

The true formulation of this approach can be represented as follows:

On a probability space $(\Omega, \Psi, P)$, let

$$
P\left(X_{i}=1\right)=P\left(X_{i}=-1\right)=1 / 2 \text {, for } i \in N
$$

Be a group of identically distributed random variables (the $X_{i}$ are said to be independent random variables). To this group, we express the sequence $\left(D_{n}, n \geq\right.$ $0)$ defined by

$$
\begin{gathered}
D_{0}=0 \\
D_{n}=\sum_{i=1}^{n} X_{i}
\end{gathered}
$$

We have $E\left(D_{n}\right)=0$ and $\operatorname{Var}\left(D_{n}\right)=n$. we can say that the sequence is a random walk [8]. We can illustrate it as a game of tossing a coin. The player gains $\$ 1$ if it comes up head and looses $\$ 1$ if tails appear. Let us start with that the player has no initial wealth $\left(D_{0}=0\right)$. His capital at time $n$ (after $n$ tosses) is $D_{n}$. If we draw the results of $N$ successive tosses, we can plot the outcomes as below: 


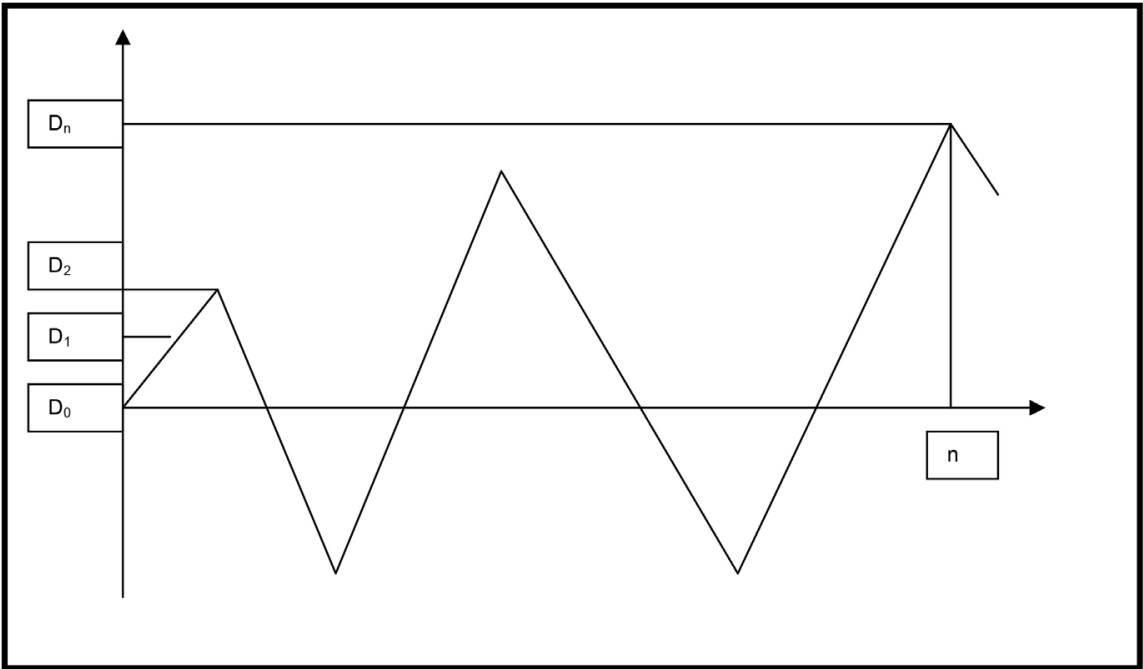

Random walk.

It is to be noted that the sequence $\left(D_{m}-D_{n}\right)$ at $m \geq n$ is independent of $\left(D_{0}, D_{1}, D_{2}, \cdots, D_{n}\right)$. Here $D_{m}-D_{n}$ has the same probability law as $D_{m-n}$ as the binomial distribution depends only on $(m-n)$.

Let us follow a two stage normalization. Let $\mathrm{N}$ be fixed. In the first stage we transform the time interval $[0, N]$ into an interval $[0,1]$ and in the second stage, we change the scale of values taken by $D_{n}$. Hence, actually we define a group of random variables bt real numbers of the form $k / N$ for $k \in N$.

$$
U_{k / N}=\frac{1}{\sqrt{N}} D_{k}
$$

We move from $U_{\frac{k}{N}}$ to $U_{\frac{k+1}{N}}$ in a very small time interval $1 / N$ by making a small displacement $\frac{1}{\sqrt{N}}$ in any of the two directions). We have

$$
E\left(U_{\frac{k}{N}}\right)=0 \text { and } \operatorname{Var}\left(U_{\frac{k}{N}}\right)=k / N
$$

The independency and stationary properties of random walk still holds.

Hence $U_{N}$ converges to a process $B$ that has continuous path (i.e. for almost all $\omega$, the mapping $t \rightarrow B_{t}(\omega)$ is continuous) and which satisfies

1) $B_{0}=0$;

2) $B_{t+\mathrm{s}}-B_{t}$ has normal distribution with $N(0, \mathrm{~s})$;

3) $B_{t+s}-B_{t}$ is independent of $B_{t(i)}-B_{t(i+1)}$ for $t_{0}<t_{1}<t_{2}<\cdots<t_{n}=t$.

Brownian motion is the only process that satisfies (1) and (3) above. To show that the distribution depends only on $\mathrm{s}$, we introduce the notation $\Delta B(t)=B(t+$ $\Delta t)-B(t)$ where $B(t)=B_{t}$ and $\Delta t>0$. The Brownian motion then satisfies

$$
\begin{gathered}
E[\Delta B(t)]=0, \operatorname{Var}[\Delta B(t)]=\Delta t \quad \text { using (2) } \\
E_{t}[\Delta B(t)]=0, \quad E_{t}\left[(\Delta B(t))^{2}\right]=\Delta t \quad \text { using (2) and (3) }
\end{gathered}
$$


where $E_{t}$ is the conditional expectation with respect to $\Psi_{t}=\sigma\left(B_{s}, s \leq t\right)$. The equality $E_{t}(\Delta B(t))=0$ can be interpreted as if the position of the Brownian motion at time $\mathrm{t}$ is known, then averahe move between time $\mathrm{t}$ and $t+\Delta t$ is zero. This property is the result of independency and Gaussian nature of Brownian motion [9].

\section{Conclusions}

Above analysis suggests that in case of low involvement products, the consumers select a product randomly from a set of such product options that fetch her similar utility. Observing the probability distribution function (pdf) and different attributes of low involvement products, the consumers would be able to derive a steady utility which is again a distribution function in mathematical context and that distribution follows the path of a particle in Brownian motion [10].

Our model would help the marketers to decide that low involvement products need to be kept places like aisles, passageways, around the check in checkout counters so that it creates agitation in customer's mind when they walk inside retail store.

\section{Conflicts of Interest}

The author declares no conflicts of interest regarding the publication of this paper.

\section{References}

[1] Kotler, P., Lilien, G.L. and Moorthy, K.S. (1992) Marketing Models. Kotler on Marketing y Marketing Insights: From $A$ to $Z$.

[2] Roberts, J.H. and Lattin, J.M. (1997) Consideration: Review of Research and Prospects for Future Insights. Journal of Marketing Research, 406-410. https://doi.org/10.1177/002224379703400309

[3] DeSarbo, W.S., Young, M.R. and Rangaswamy, A. (1997) A Parametric Multidimensional Unfolding Procedure for Incomplete Nonmetric Preference/Choice Set Data in Marketing Research. Journal of Marketing Research, 499-516. https://doi.org/10.1177/002224379703400407

[4] Gupta, S. and Zeithaml, V. (2006) Customer Metrics and Their Impact on Financial Performance. Marketing Science, 25, 718-739. https://doi.org/10.1287/mksc.1060.0221

[5] Dwyer, F.R. (1997) Customer Lifetime Valuation to Support Marketing Decision Making. Journal of Interactive Marketing, 11, 6-13.

[6] Elheridge, A. (2002) A Course in Financial Calculus. Cambridge.

[7] Lin, K.Y. and Sibdari, S.Y. (2009) Dynamic Price Competition with Discretecustomer Choices. European Journal of Operational Research, 197, 969-980. https://doi.org/10.1016/j.ejor.2007.12.040

[8] Osborne, M.F.M. (1959) Brownian Motion in the Stock Market. Operations Research, 7, 145-173. https://doi.org/10.1287/opre.7.2.145

[9] Tremblay, C.H., Tremblay, M.J. and Tremblay, V.J. (2011) A General CournotBertrand Model with Homogeneous Goods. Theoretical Economics Letters, 1, 38. 
https://doi.org/10.4236/tel.2011.12009

[10] Soliman, A. and Obi, J. (2017) Bank Capitalisation and Stock Market Growth: Theoretical Model and Empirical Evidence. Theoretical Economics Letters, 7, 17471760. https://doi.org/10.4236/tel.2017.76118 